\title{
Experimentelle Untersuchungen zum Stockwachstum und zur Medusenbildung bei dem marinen Hydrozoon Eirene viridula
}

\author{
M. Bierbach \& D. K. Hofmann \\ Institut für Entwicklungsphysiologie der Universität Köln; \\ Köln, Bundesrepublik Deutschland
}

\begin{abstract}
Experimental studies on colony growth and on budding of medusae in the marine hydroid Eirene viridula. The development of both slide-grown and non-substrate bound colonies of $E$. viridula (Thecata-Leptomedusae) ranging in size from 1 to 50 hydranths was investigated under various temperature conditions. The majority of slide-grown colonies reached a larger final size than non-substrate bound ones, in $20^{\circ}, 25^{\circ}$ and $29^{\circ} \mathrm{C}$. Raising the temperature did not stimulate propagation of hydranths as expected. Most of the colonies transferred to $25^{\circ}$ or $29^{\circ} \mathrm{C}$ finally were even smaller than those reared at $20^{\circ} \mathrm{C}$. This was partially due to resorption of several hydranths about 9 days after the temperature rise; the influence of "physiological competition" between development of new hydranths and budding of medusae on colony growth is discussed. Transfer from higher to lower temperatures affected colony growth negatively. Raising the temperature from $20^{\circ}$ to $25^{\circ}$ or $29^{\circ} \mathrm{C}$ initiated formation of gonozooids from the distal part of hydranth stalks and development of medusa buds in both types of colonies. With the exception of slide-grown colonies transferred to $25^{\circ} \mathrm{C}$, also young medusae were budded off. There was a remarkable coincidence in predominance of colony growth in slide-grown colonies and of medusa budding in non-substrate bound cultures. In the latter, medusa buds developed 1 to 2 days earlier. Most buds did not differentiate into liberated medusae, but were resorbed. Transformation of medusa buds into hydranths was not observed. In the clone of $E$. viridula, onset of medusa budding did not depend on a "minimal colony size". Even single hydranths were able to produce medusa buds after transfer to higher temperatures; budded off medusae were recorded from non-substrate bound colonies with an initial size of 3 hydranths. In slide-grown cultures, medusa buds developed into colonies with an initial size of only 3 hydranths. No hydranth propagation prior to medusa budding occurred in these cases. After raising temperature from $25^{\circ}$ to $29^{\circ} \mathrm{C}$ medusa buds were observed in nonsubstrate bound colonies only; a small number of medusae were budded off from some of these colonies. Lowering the temperature from $29^{\circ}$ or $25^{\circ}$ to $20^{\circ} \mathrm{C}$ caused resorption of existing medusa buds. In several non-substrate bound colonies, transfer from $29^{\circ}$ to $25^{\circ} \mathrm{C}$ induced development of gonozooids with medusa buds and, in some cases, of young medusae. Incubation with the alkylating cytostatic Trenimon and transfer from $20^{\circ}$ to $25^{\circ} \mathrm{C}$ caused irreversible resorption of all hydranths when $4 \times 10^{-2} \mathrm{mg} / \mathrm{ml}$ were administered for 10 mins. Thereafter, only development of stolonial structures was observed. With one exception, the colonies treated with $4 \times 10^{-3} \mathrm{mg} / \mathrm{ml}$, and all others submitted to $4 \times 10^{-4} \mathrm{mg}$ TrENIMON/ml were able to produce new hydranths and also medusa buds; some of the colonies first had to overcome a degressive phase. Treatment with $4 \times 10^{-2} \mathrm{mg}$ destroyed all interstitial cells (I-cells). Incubation with $1 \times 10^{-3}$ or $1 \times 10^{-4} \mathrm{mg} / \mathrm{ml}$ left the $I$-cells at least partially intact. It is concluded that I-cells are indispensable for hydranth and medusan morphogenesis in E. viridula.
\end{abstract}




\section{EINLEITUNG}

Die Medusenbildung bei Eirene viridula und besonders die Frage ihrer experimentellen Auslösbarkeit hatte GünzL $(1959,1964)$ untersucht. Er ermittelte, daß Polypenstöcke durch Überführen aus niedrigen in höhere Temperaturen zur Medusenbildung veranlaßt werden konnten. Die Entstehung von Medusenknospen war von einer Stockmindestgröße zum Zeitpunkt des Temperaturwechsels abhängig, allerdings ergab sich bei den vegetativen Abkömmlingen von Zuchtstöcken unterschiedlicher Größe eine nicht unerhebliche Variabilität in der Reaktion auf positive Temperaturänderungen. Somit war nach den Untersudhungen von ReIsinger (1957) an Craspedacusta sowerbyi und WERNER $(1958,1961,1968)$ an Ratblea octopunktata, Bougainvillia superciliaris und Eucheilota maculata sowie der von GüNzL (1964) ebenfalls studierten Dipurena reesi für eine weitere $H y d r o i d e n-S p e z i e s$ die Umgebungstemperatur als dominierender Faktor für die Auslösung der Medusenbildung experimentell belegt worden.

In der vorliegenden Arbeit untersuchten wir das Verhalten von stolonial-flächig wachsenden und außerdem von freischwimmend gehaltenen Stöcken mit kleinen $\mathrm{Hy}$ dranthenzahlen bei Zucht unter konstanten Temperaturbedingungen sowie nach positivem oder negativem Temperaturwechsel unterschiedlichen Ausmaßes. Von Interesse waren dabei sowohl das Stockwachstum durch vegetative Propagation von Polypen als auch die Bildung von Medusenknospen und Medusen unter den jeweiligen Bedingungen. Im Anschluß daran wurden Versuche zu einer Analyse der zytologischen Voraussetzungen der Blastostyl- und Medusenknospenbildung unternommen.

\section{VERSUCHSTIERE UND METHODEN}

Als Ausgangsmaterial für die Zuchten stand uns ein freischwimmender Stock von Eirene viridula zur Verfügung, den uns Herr Dr. B. WERner, Biologische Anstalt Helgoland, 1968 freundlicherweise überlassen hatte. Sämtliche Tochterstöcke wurden durch Vereinzelung aus diesem Stock und seinen rein vegetativen Abkömmlingen gewonnen. Sessile Stöcke züchteten wir ausnahmslos auf Objektträgern, die einzeln in Boverischalen mit ca. $150 \mathrm{ml}$ pasteurisiertem, natürlichem Meerwasser (Nordseedichte) gehalten wurden. Freischwimmende Stöcke wurden ebenfalls einzeln in Boverischalen gehalten; diese Wuchsform ist leicht dadurch zu erzielen (vgl. HaUENSCHILD 1954 für Hydractinia ecbinata), daß man zusammen mit Stolonenmaterial isolierte Polypen daran hindert, sich am Schalenboden festzusetzen. Unter diesen Bedingungen kugelt sich das Material ab und bildet einen kleinen, freischwimmenden Stock. Gefüttert wurde zweimal wöchentlich mit Artemia-Nauplien; häufigere Fütterung hatte sich ungünstig auf das Stockwachstum ausgewirkt. Etwa 3 Stunden nach der Fütterung wurden die Stöcke durch "Ausblasen." mit einer Pipette gründlich von Futterresten befreit, danach erfolgte Wasserwechsel mit gemäß der jeweiligen Versuchsanordnung temperiertem Meerwasser.

Die Temperaturversuche wurden bei konstant $20^{\circ} \mathrm{C}$ (Anzuchttemperatur), $25^{\circ}$ und $29^{\circ} \mathrm{C}$ in Klimakammern bzw. Thermostaten $\left(29^{\circ} \mathrm{C}\right.$-Stufe) durchgeführt. Zur Vermeidung von Temperaturschwankungen wurden die Kulturschalen der $29^{\circ} \mathrm{C}$-Stufe für 
die Dauer der Fütterung und der täglichen Kontrollen in ein Wasserbad gestellt, das durch einen LAUDA-Thermostaten im Durchfluß-System auf $29^{\circ} \mathrm{C}$ temperiert wurde. Für die Versuche mit positivem Temperaturwechsel von $20^{\circ}$ nach $25^{\circ} \mathrm{C}$ bzw. $29^{\circ} \mathrm{C}$ setzten wir je einen Stodk der Ausgangsgröße (A): 1, 2, 3, 4, 5, 6, 7, 8, 9, 10, 12, 14, 16, $18,20,25,30,35,40$ und 50 Polypen ein (gezählt wurden die ausdifferenzierten Hydranthen). Ferner wurde jeweils eine Wiederholungsserie gleichen Umfanges angesetzt. Die Beobachtungszeit betrug 17 Tage, vom Temperaturwechsel an gerechnet. Für die Auswertung wurden beide Serien zusammengefaßt.

Bei den weiteren Versuchen im einzelnen etwas abweichende Stockanzahlen und Beobachtungsdauer werden im jeweiligen Abschnitt vermerkt.

Falls nicht anders angegeben, erfolgte die Kontrolle des Polypenzuwachses, der angelegten Medusenknospen sowie der freigewordenen Medusen täglich, letztere wurden sofort aus den Zuchtschalen entfernt. Als Beginn der Medusenknospung wertete

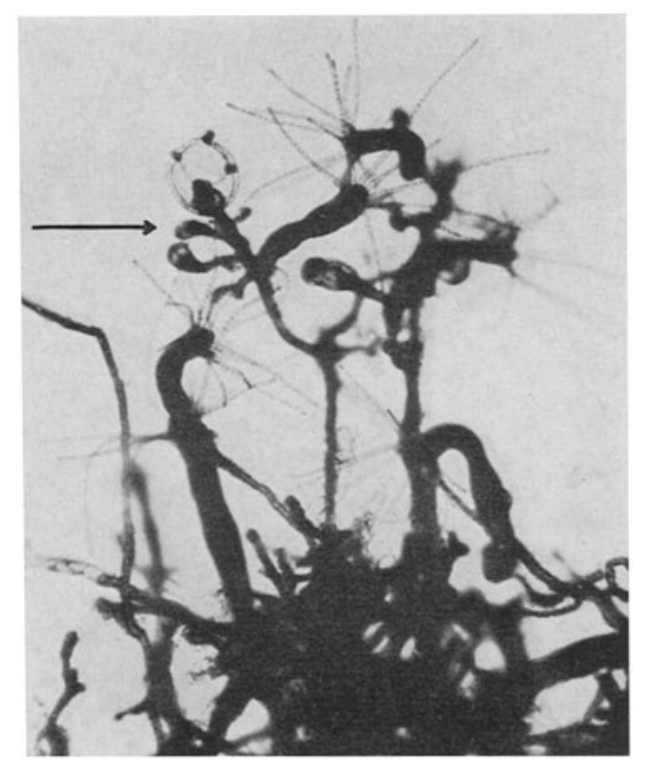

Abb. 1: Eirene viridula. Lebendaufnahme einer freischwimmend gehaltenen Polypenkolonie mit verschieden weit entwickelten Medusenknospen. (Ausschnittvergrößerung 16:1)

GüNZL bereits die Entstehung der Blastostyle an der Basis der Hydranthen. In unserer Untersuchung wurden Medusenknospen dann als solche registriert, wenn die Glockenhöhle sichtbar war (Abb, 1). "Dauerkulturen" wurden 2 Monate lang bei $25^{\circ}$ und $29^{\circ} \mathrm{C}$ gehalten und regelmäßig kontrolliert; aus diesen. Ansätzen wurden die Stöcke für den Umsetzversuch von $25^{\circ}$ nach $29^{\circ} \mathrm{C}$ und für die Versuche mit negativem Temperaturwechsel vereinzelt.

Die angewendeten zytologischen Untersuchungsmethoden werden im entsprechenden Textabschnitt angegeben. 


\section{ERGEBNISSE}

\section{Stockentwicklung bei $20^{\circ}$}

Auf Objektträgern stolonial-flächig wachsende Stöcke zeigten innerhalb des Beobachtungszeitraumes von 17 Tagen eine monotone Größenzunahme um das 2- bis 7 fache der Ausgangsgröße; Degressionsphasen traten nicht auf.

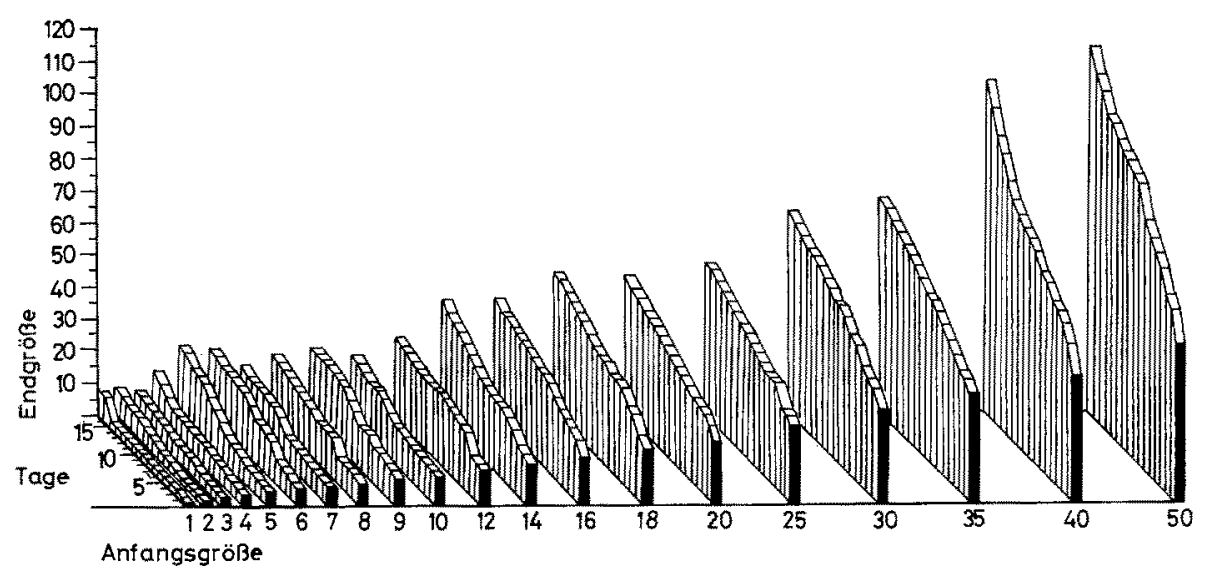

Abb. 2: Eirene viridula. Stockwachstum bei 20 freischwimmenden Stöcken bei $20^{\circ} \mathrm{C}$ während 17 Tagen (Wiederholungsversuch). Gezählt wurden die ausdifferenzierten Polypen

Freischwimmende Stöcke nahmen im selben Zeitraum um das 2- bis 8 fache ihrer Ausgangsgröße zu; Degressionsphasen traten nicht auf. Der Verlauf des Stodkwachstums ist für diese Kategorie in Abbildung 2 dreidimensional in absoluten Werten dargestellt.

Der paarweise Vergleich zwischen sessilen und freischwimmenden Stöcken gleicher Ausgangsgröße ergab in 34 von 40 Vergleichspaaren einen größeren Zuwachs bei den sessilen Stöcken $(+4$ bis +44$)$. Blastostyle, Medusenknospen oder freie Medusen wurden unter diesen Temperaturbedingungen nie beobachtet.

\section{Stockentwicklung nach Umsetzen in höhere Temperaturen}

\section{Stockentwicklung nach Umsetzen won $20^{\circ}$ nach $25^{\circ} \mathrm{C}$}

Sessile Stöcke zeigten nach dem Temperaturwechsel im Verlauf von 17 Tagen einen $\mathrm{Zuwachs} \mathrm{um} \mathrm{das} \mathrm{1,5-} \mathrm{bis} 14$ fache. Monotone Größenzunahme erfolgte bei einigen kleineren Stöcken, alle übrigen ließen diskontinuierlichen Zuwachs mit Stockdegression infolge Einschmelzens von Polypen am 9. Tag nach Temperaturwechsel erkennen. Die bei Versuchsende erreichte Hydranthenzahl war bei Stöcken ab (A) 18 stets kleiner als 
bei den entsprechenden bei $20^{\circ} \mathrm{C}$ gehaltenen Kolonien. Stöcke kleinerer Größenklassen verhielten sich unterschiedlich.

Nur 11 der insgesamt 40 sessilen Stöcke bildeten unter diesen Temperaturbedingungen Medusenknospen aus. Die ersten Medusenknospen wurden am 5. Tag nach Temperaturwechsel registriert, der späteste Knospungsbeginn lag am 14. Tag. Manche Stöcke hatten nur eine Knospungsphase, andere deren zwei. - Freie Medusen traten nicht auf (vgl. Abb. $4 a$ und 6).

Freischwimmende Stöcke zeigten fast ausnahmslos einen diskontinuierlichen Wachstumsverlauf mit Stockdegression bzw. Wachstumsstagnation am 9. Tag nach

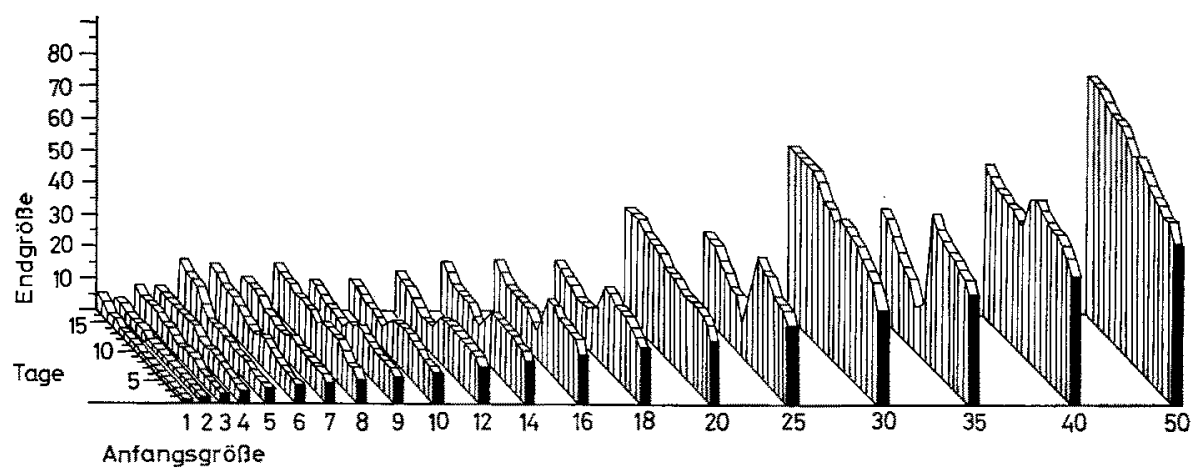

Abb. 3: Eirene viridula. Stockwachstum bei 20 freischwimmenden Stöcken während 17 Tagen nach Umsetzen von $20^{\circ}$ nach $25^{\circ} \mathrm{C}$ (Wiederholungsversuch). Gezählt wurden die ausdifferenzierten Polypen

Temperaturwechsel. Zu Versuchsende war in 32 von 40 Fällen die erreichte Polypenzahl erheblich kleiner als bei den entsprechenden bei $20^{\circ} \mathrm{C}$ gehaltenen Stödken $(-2$ bis -36). Der Verlauf des Stockwachstums dieser Kategorie ist in Abbildung 3 dreidimensional in absoluten Werten dargestellt.

35 von 40 Stöcken bildeten Medusenknospen; diese traten bei der Hälfte der Stöcke bereits 3 Tage nach dem Temperaturwechsel auf, bei den übrigen zwischen dem 4. und 15. Tag. Die Mehrzahl zeigte nur eine bis zum Versuchsende währende Knospungsphase. Die kleinsten Stöcke mit (A) 2 besaßen bei Knospungsbeginn am 11. Tag erst 3 Polypen. Bei 26 Stöcken dieser Kategorie traten freie Medusen auf, und zwar erstmals am 5. Tag. Der kleinste Stock besaß zu diesem Zeitpunkt 6 Polypen.

Kolonien der beiden Wuchsformen zeigten nach Temperaturwechsel von $20^{\circ}$ nach $25^{\circ} \mathrm{C}$ unterschiedliches Verhalten sowohl hinsichtlich des Stockzuwachses als auch der Medusenbildung: bei 34 von 40 Vergleichspaaren lagen die Endgrößen sessiler Stöcke höher als die der freischwimmenden $(+3$ bis +41$)$. Während nur wenige sessile Stöcke in geringem Umfange Medusenknospen und überhaupt keine freien Medusen erzeugten, war diesbezüglich die Aktivität freischwimmender Kolonien ungleich höher: fast alle Stöcke bildeten Medusenknospen und die Mehrzahl auch freie Medusen (Abb. 4a, 5a und 6). 


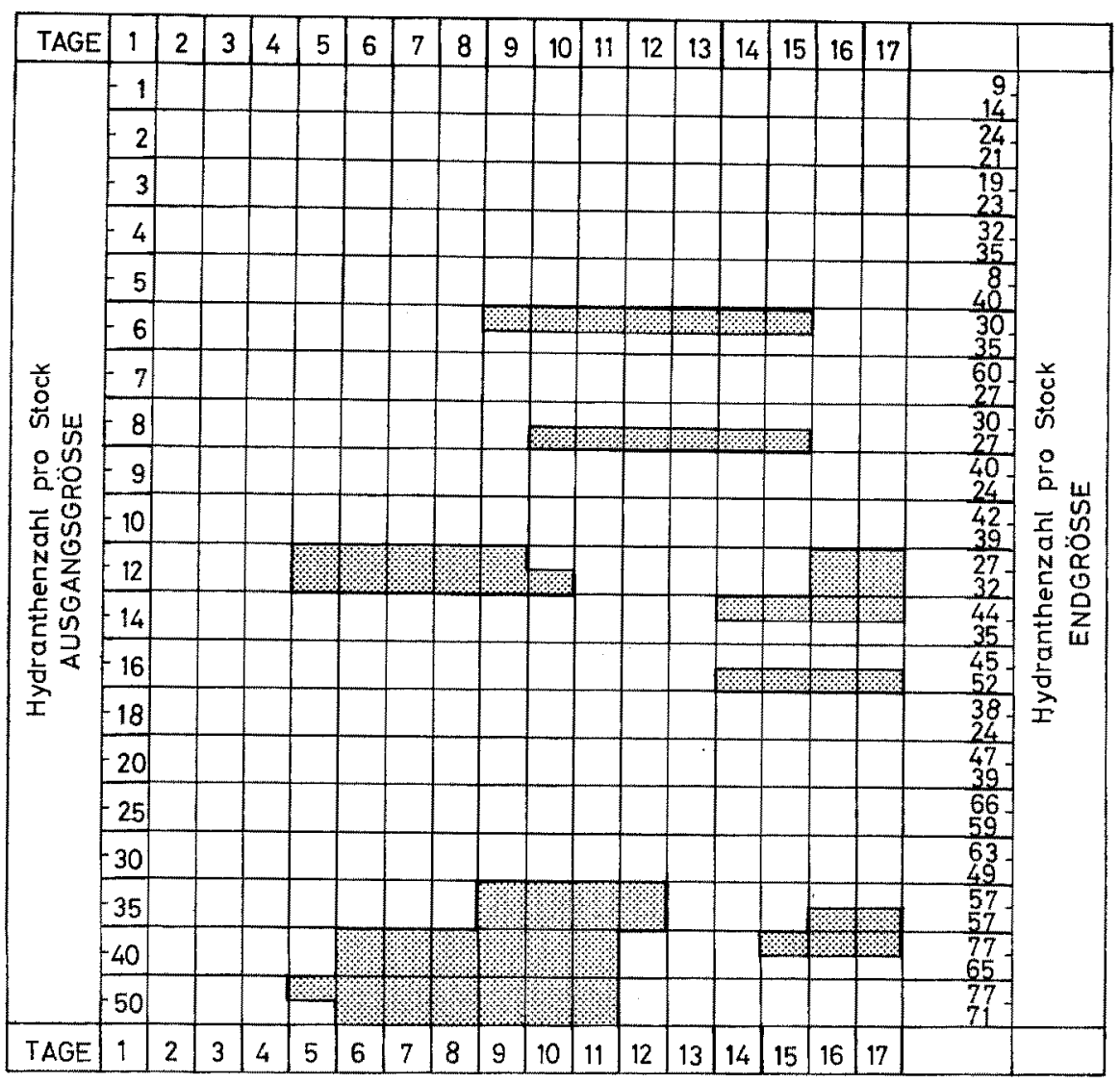

Abb. 4a: Eirene viridula. Phasen der Medusenknospen- und Medusenbildung sowie Stockentwicklung bei 40 sessilen Polypenstöcken im Verlauf von 17 Tagen nach Umsetzen von $20^{\circ}$ nach $25^{\circ} \mathrm{C}$ (H.aupt- und Wiederholungsversuch zusammengefaßt). Gerasterte Flächen: Medusenknospenbildung; schwarz angelegte Flächen: Medusenbildung

Stockentwicklung nach Umsetzen von $20^{\circ}$ nach $29^{\circ} \mathrm{C}$

Sessile Stöcke wiesen in 30 Fällen diskontinuierliches Wachstum mit Stodkdegression am 9. Tag auf, die übrigen 10 wuchsen monoton. Teils erfolgte dabei Hydranthenvermehrung um das 2- bis 13 fache, teils aber auch Größenabnahme bis unter die Ausgangsgröße.

Verglichen mit den bei $20^{\circ} \mathrm{C}$ aufgezogenen Stöcken derselben Wuchsform waren von den 40 eingesetzten Stöcken 27 kleiner als jene (Extremwert: - 50). Auch gegenüber der Endgröße der nach $25^{\circ} \mathrm{C}$ umgesetzten sessilen Kolonien waren 29 Stöcke kleiner (Extremwert: - 30). Insgesamt 18 Stöcke bildeten zwischen dem 5. und 16. Tag Medusenknospen. Der Kleinste mit (A) 2 besaß zu diesem Zeitpunkt 18 Polypen; (A) 3 bildete, ohne größer geworden zu sein, am 5. Tag die erste Medusenknospe. 


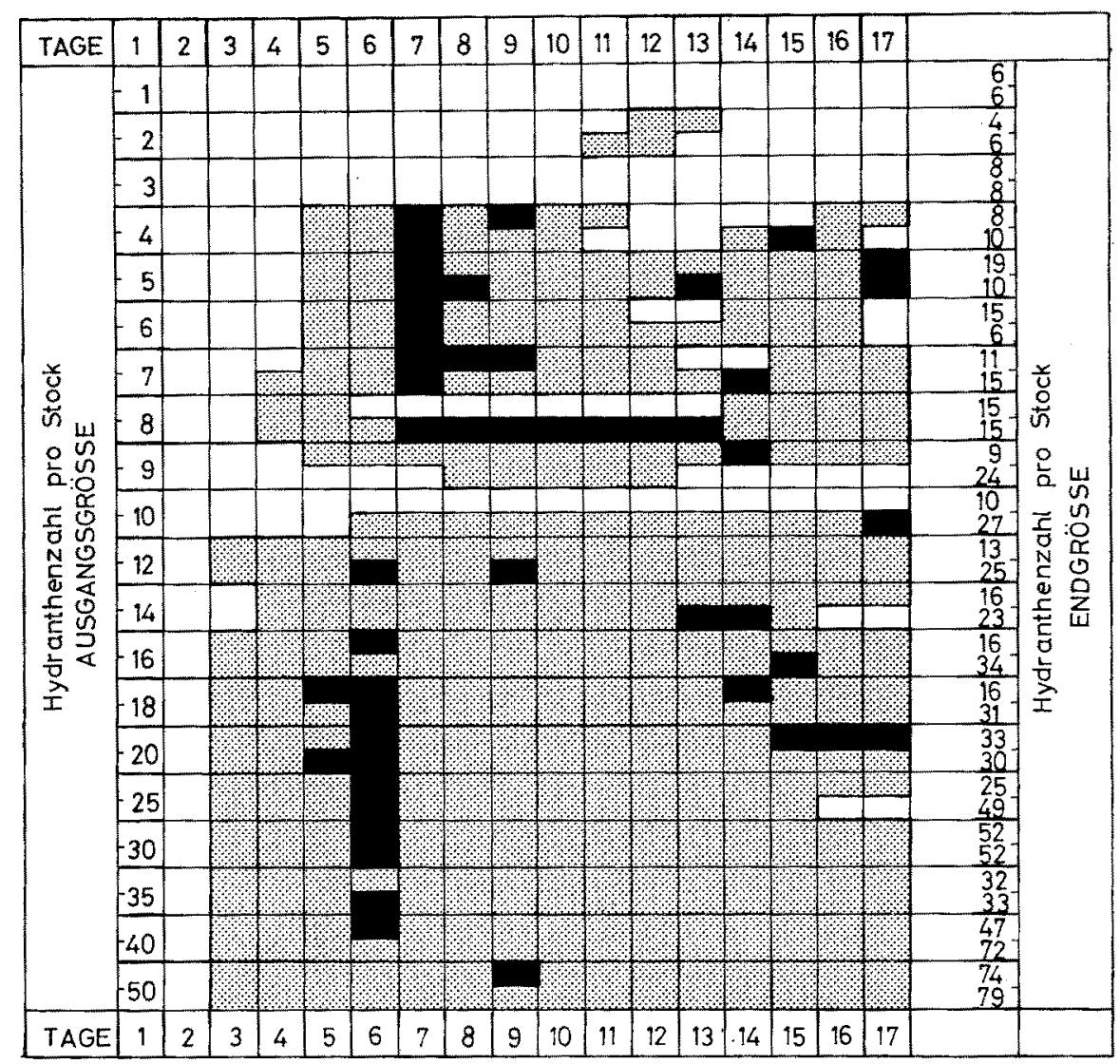

Abb. 5a: Eirene viridula. Phasen der Medusenknospen- und Medusenbildung sowie Stockeutwidklung bei 40 freischwimmenden Polypenstöcken im Verlauf von 17 Tagen nach Umsetzen von $20^{\circ}$ nach $25^{\circ} \mathrm{C}$ (Haupt- und Wiederholungsversuch zusammengefaßt). Gerasterte Flächen: Medusenknospenbildung; schwarz angelegte Flächen: Medusenbildung

Gegenüber den nach $25^{\circ} \mathrm{C}$ umgesetzten sessilen Stöcken erhöhte sich die Zahl der medusenknospenbildenden Stödke um 50\%, überdies war die Tendenz zur Verlängerung der Knospungsphase und Steigerung der Knospenanzahl mit wachsender Stockgröße unverkennbar. Die Zahl freier Medusen blieb jedoch gering: nur 3 Stöcke bildeten je eine Meduse. Freischwimmende Stöcke vermehrten in 17 Tagen zum Teil die Hydranthenzahl um das 2- bis 3 fache, teilweise verringerte sich jedoch die Anzahl der Polypen. Mit einer Ausnahme trat Wachstumsstagnation bzw. Stockdegression am 9. Tag nach Temperaturwechsel ein, die sich in einigen Fällen in den folgenden Tagen noch verstärkte. Im Vergleich zu den bei $20^{\circ} \mathrm{C}$ gehaltenen freischwimmenden Stöcken erreichten 33 eine geringere Endgröße (Extremwert: - 31). Gegenüber den nach $25^{\circ} \mathrm{C}$ umgesetzten Kolonien wies die Hälfte der Stöcke kleinere Endgrößen auf.

Mit einer Ausnahme bildeten alle Stöcke zwischen dem 4. und 14. Tag Medusenknospen, wobei die meisten nur eine Knospungsphase zeigten. Der kleinste "Stock" 


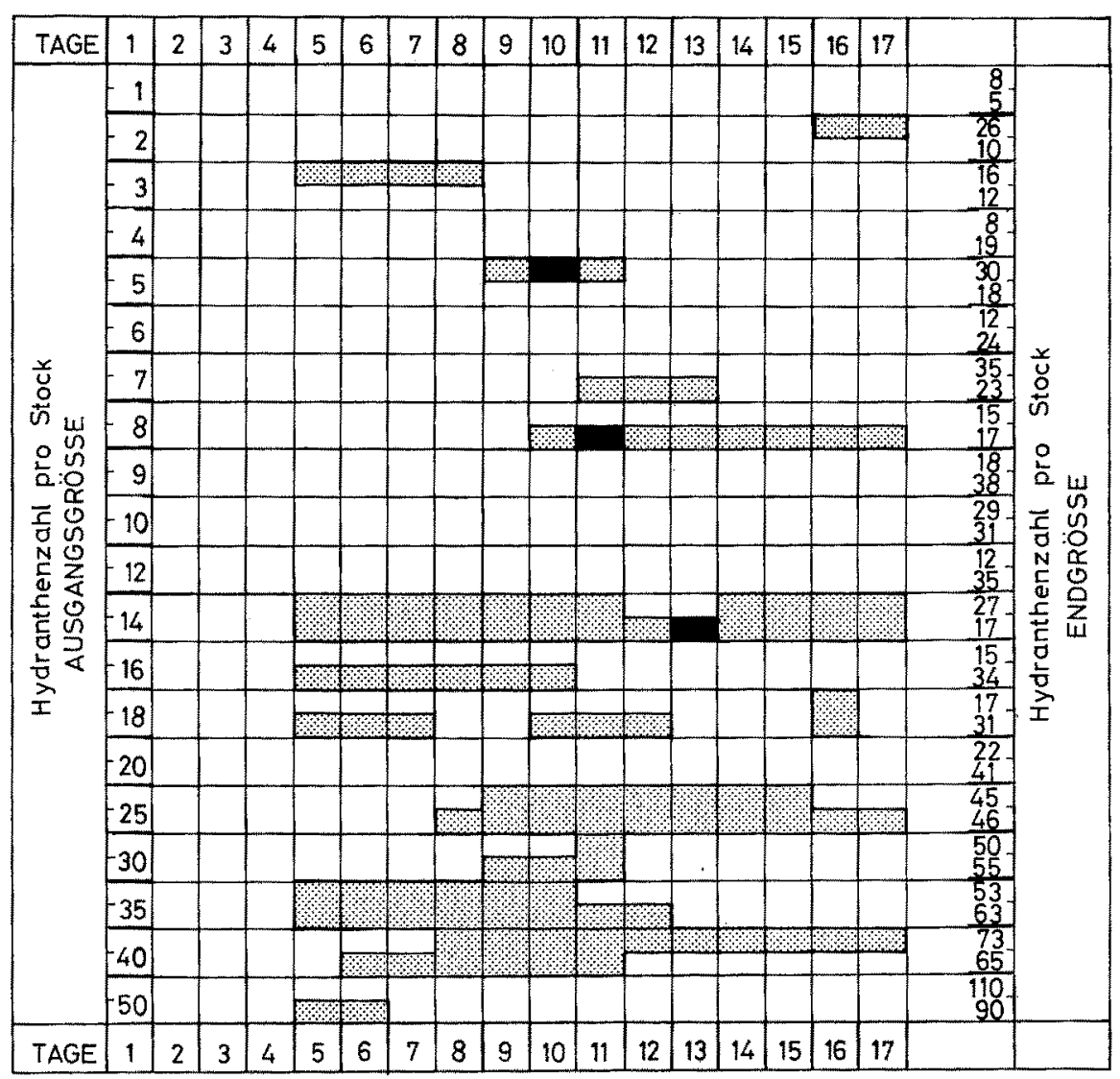

Abb. 4b: Eirene viridula. Phasen der Medusenknospen- und Medusenbildung sowie Stockentwicklung bei 40 sessilen Polypenstöcken im Verlauf von 17 Tagen nach Umsetzen von $20^{\circ}$ nach $29^{\circ} \mathrm{C}$ (Haupt- und Wiederbolungsversuch zusammengefaßt. Kennzeichnung wie in Abb. 4a)

besaß zu Beginn der Knospenbildung einen einzigen Polypen. 23 medusenknospenbildende Stöcke erzeugten auch freie Medusen (Abb. 5b). Gegenüber den nach $25^{\circ} \mathrm{C}$ umgesetzten freischwimmenden Stöcken war lediglich eine Verzögerung des Beginns der Medusenknospung um einen Tag $z u$ bemerken (Abb. 5a, b).

Zwischen den Stöcken der beiden Wuchsformen ergaben sich nach Umsetzen von $20^{\circ}$ nach $29^{\circ} \mathrm{C}$ ebenfalls Unterschiede sowohl hinsichtlich des Stockzuwachses als auch der Medusenknospen- und Medusenbildung: 27 der 40 sessilen Stöcke waren bei Versuchsende größer als die entsprechenden freischwimmenden. Sessile und freischwimmende Stöcke reagierten zwar auf den Temperaturwechsel gleichermaßen mit Bildung von Medusenknospen und Medusen, in quantitativer Hinsicht dominierte jedoch hier die freischwimmende Wuchsform: die Zahl der medusenknospenbildenden Kolonien war doppelt so groß wie die der sessilen, die Anzahl der freie Medusen bildenden Stöcke war sogar $8 \mathrm{mal}$ so groß (vgl. Abb. 6). 


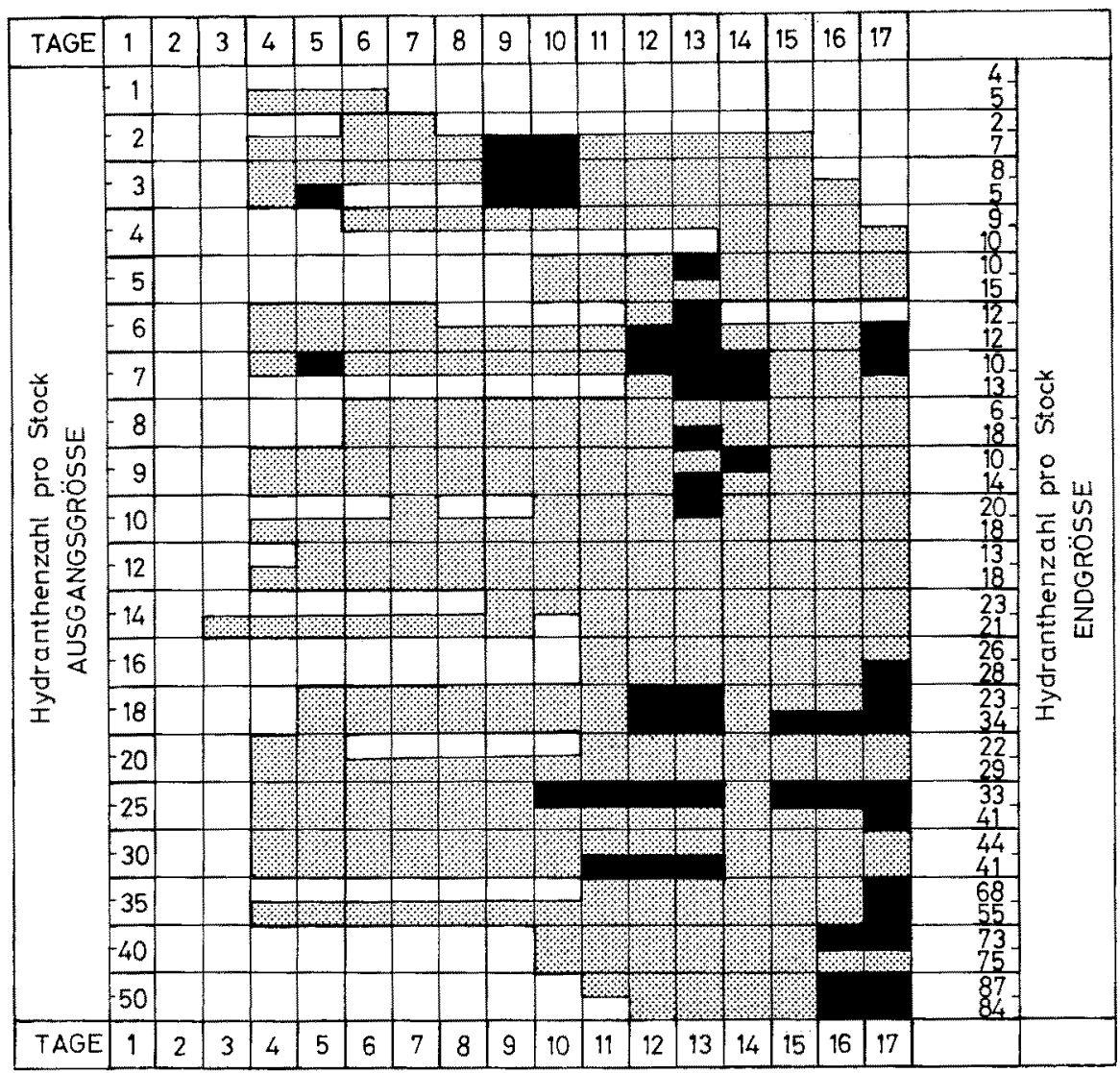

Abb. 5b: Eirene viridula. Phasen der Medusenknospen- und Medusenbildung sowie Stockentwicklung bei 40 freischwimmenden Polypenstöcken im Verlauf von 17 Tagen nach Umsetzen von $20^{\circ}$ nach $29^{\circ} \mathrm{C}$. (Haupt- und Wiederholungsversuch zusammengefaßt. Kennzeichnung wie in $\mathrm{Abb} .5 \mathrm{a})$

\section{Stockentwicklung von "Dauerkulturen " bei $25^{\circ}$ und $29^{\circ} \mathrm{C}$}

Für die Temperaturwechselversuche von $25^{\circ}$ nach $29^{\circ} \mathrm{C}$ sowie für die Versuche mit negativem Temperatursprung wurden Stöcke benötigt, bei denen die Wirkungen des beim Einsetzen in die höheren Anzuchttemperaturen unumgänglichen Temperaturwechsels abgeklungen waren. Als mutmaßlichen Zeitraum hierfür nahmen wir, unter Berücksichtigung der Erfahrungen Günzls, zwei Monate an.

Ober die Stockentwicklung in den ersten 17 Tagen nach positivem Temperaturwechsel von $20^{\circ}$ nach $25^{\circ} \mathrm{C}$ bzw. $29^{\circ} \mathrm{C}$ wurden die Ergebnisse zuvor bereits niedergelegt. Die nach diesem Zeitraum 2- bis $4 \mathrm{mal}$ wöchentlich vorgenommenen Kontrollen zeigten, daß unter $25^{\circ} \mathrm{C}$-Bedingungen nur bei freischwimmenden Stödken bis zum Versuchsende, wenn auch nur in geringem Umfang, noch Medusenknospen auftraten. 
Freie Medusen wurden in der zweiten Hälfte der Beobachtungszeit nicht mehr gebildet. Auch unter $29^{\circ} \mathrm{C}$-Bedingungen waren lediglich bei den freischwimmenden Stöcken, vor allem bei solchen mit großen Hydranthenanzahlen, im zweiten Monat der Beobachtungszeit noch Medusenknospen, nicht aber freie Medusen zu sehen. Zu Versuchsende fanden sich auch keine Knospen mehr.

\section{Stockentwicklung nach Umsetzen von $25^{\circ}$ nach $29^{\circ} \mathrm{C}$}

Für diesen Versuch stand je eine vollständige Serie sessiler und freischwimmender Stöcke der Ausgangsgrößen 1 bis 50 Polypen zur Verfügung. Sie wurden „Dauerkulturen" (siehe pp. 65 und 71) entnommen und besaßen zur Zeit des Versuchsbeginnes keine Medusenknospen. Die Auszählung der Stöcke erfolgte 16 Tage lang jeden zweiten Tag. Sessile Stöcke zeigten im Vergleich mit den zuvor beschriebenen Temperaturwechselversuchen ein geringeres Stockwachstum; Stockdegressionen traten jedoch nicht auf. Medusenknospen oder Medusen wurden unter diesen Bedingungen nicht gebildet.

Freischwimmende Stöcke wuchsen nach Temperaturwechsel ebenfalls nur langsam; die Werte entsprachen den für die sessilen Stödke dieser Versuchsreihe ermittelten. Stockdegressionen traten nicht auf. Hingegen wurden bei 14 der 20 Stöcke die Bildung von Medusenknospen und außerdem bei 8 Stöcken dieser Gruppe die Entstehung freier Medusen beobachtet.

\section{Stockentwicklung nach Umsetzen in tiefere Temperaturen Stockentwicklung nach Umsetzen von $25^{\circ}$ nach $20^{\circ} \mathrm{C}$}

Es wurde je eine Serie von 16 sessilen und 16 freischwimmenden Stöcken aus

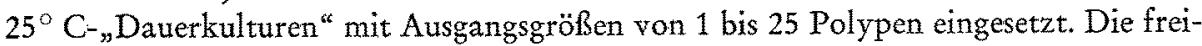
schwimmenden Kolonien besaßen z. T. noch Medusenknospen. Die Auszählung erfolgte 16 Tage lang jeden zweiten Tag. Alle sessilen Stöcke zeigten ein stark beeinträchtigtes, diskontinuierliches Stockwachstum mit Stockdegression zwischen dem 7. und 9. Tag, in deren Verlauf bei den kleinsten Stöcken alle Hydranthen zeitweilig eingeschmolzen wurden. Medusenknospen oder Medusen entstanden unter diesen Bedingungen nicht.

10 der 16 freischwimmenden Stöcke zeigten monotone Größenzunahme, die übrigen unterlagen einer Stockdegression zwischen dem 9. und 14. Tag. Medusenknospen oder Medusen wurden nicht beobachtet. Bei Versuchsbeginn noch vorhandene Medusenknospen wurden innerhalb von 24 Stunden nach Temperaturwechsel eingeschmolzen.

\section{Stockentwicklung nach Umsetzen von $29^{\circ}$ nach $20^{\circ} \mathrm{C}$}

Es wurden 18 sessile Stödke mit (A) 1 bis 35 Polypen und 15 freischwimmende Stöcke mit (A) 1 bis 20 Polypen, jeweils aus $29^{\circ} \mathrm{C}$ „,Dauerkulturen“ eingesetzt. Die Auszählung erfolgte 15 Tage lang jeden zweiten Tag. 


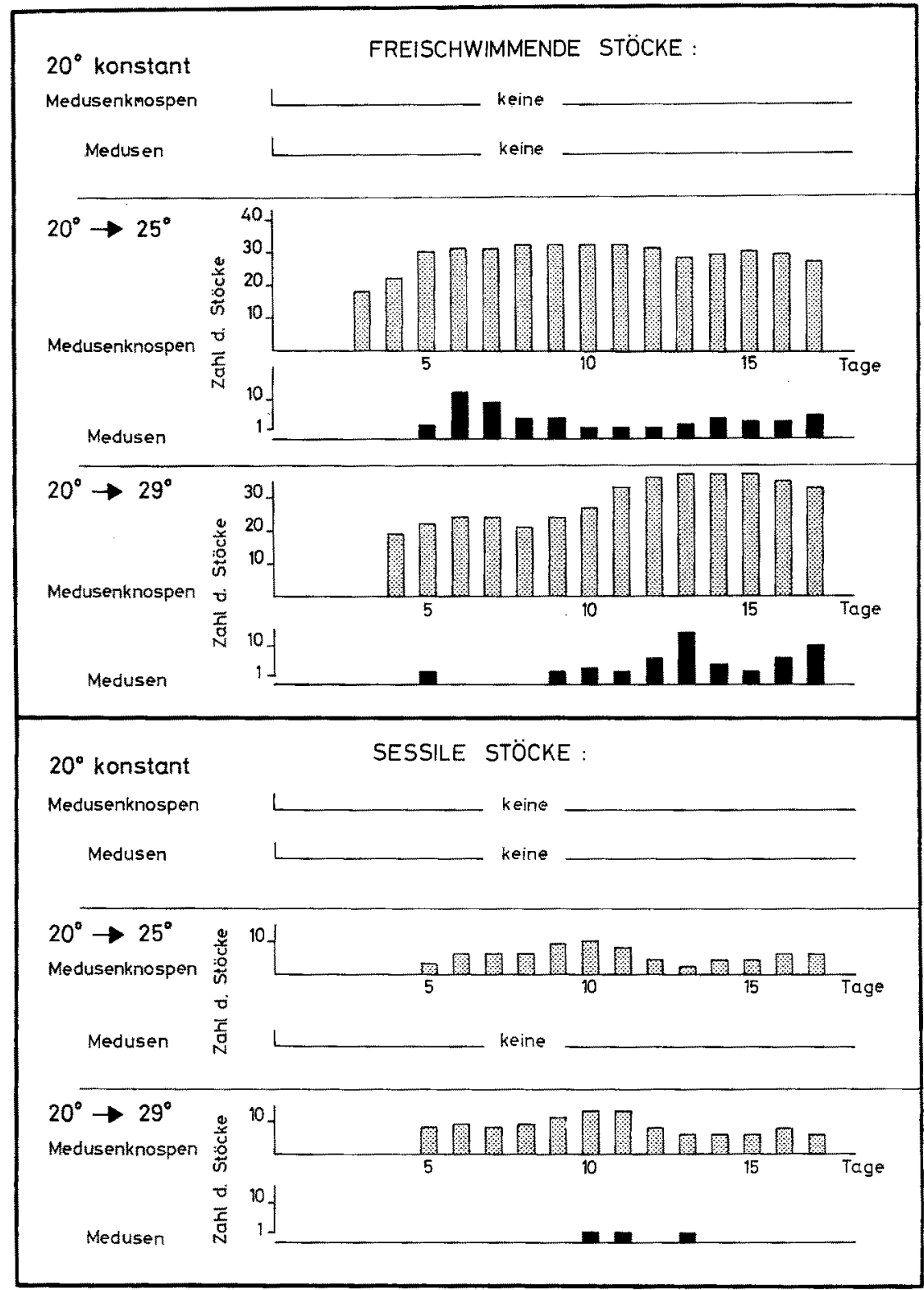

Abb. 6: Eirene viridula. Anzahl der Medusenknospen und Medusen bildenden Polypenstöcke im Verlauf von 17 Tagen. Vergleich der Versuchsglieder. (Haupt- und Wiederholungsversuche jewreils zusammengefaßt) 
Bei sessilen Stöcken war monotone Größenzunahme ohne Stockdegression festzustellen. Medusenknospen oder Medusen wurden nicht gebildet. 13 der 15 freischwimmenden Stöcke zeigten bereits zwischen dem 4. und dem 8. Tag nach Temperaturwechsel Degression bzw. Stagnation, danach bis zum Ende der Beobachtungszeit geringen Stockzuwachs. $\mathrm{Zu}$ verschiedenen Zeitpunkten nach dem Temperaturwechsel waren bei Polypen von 12 Stöcken abnorme Tentakelverkürzungen oder Verlängerungen $\mathrm{zu}$ bemerken. Medusenknospen oder Medusen wurden nicht gebildet.

\section{Stockentwicklung nach Umsetzen von $29^{\circ}$ nach $25^{\circ} \mathrm{C}$}

Für den Versuchsansatz standen 20 sessile Stöcke mit (A) 1 bis 35 Polypen und 15 freischwimmende Stöcke mit (A) 1 bis 20 Polypen aus $29^{\circ} \mathrm{C}-$ „,Dauerkulturen“ zur Verfügung, die keine Medusenknospen besaßen. Die Auszählung erfolgte 15 Tage lang jeden zweiten Tag. Alle sessilen Stöcke wuchsen monoton, Degressionserscheinungen zeigten sich nicht. Es entstanden keine Medusenknospen oder Medusen. Bei 12 freischwimmenden Stöcken war der Zuwachs monoton, bei den restlichen 3 Kolonien wurde am 11. Tag nach Temperaturwechsel eine Stockdegression festgestellt. Im Gegensatz zu den anderen Versuchen mit negativer Temperaturänderung wurden nach dem Umsetzen von $29^{\circ}$ nach $25^{\circ} \mathrm{C}$ bei 9 der freischwimmenden Stöcke vom 4. Tag an Medusenknospen beobachtet. Bei 5 Stöcken wurden zwischen dem 6. und dem 15. Tag auch freie Medusen gebildet. Dies entspricht dem Resultat des Versuches mit positiver Temperaturänderung von $25^{\circ}$ nach $29^{\circ} \mathrm{C}$.

\section{Untersuchung der zytologischen Basis der Medusenbildung}

Experimentell-zytologische Untersuchungen zur Medusenbildung, die GüNZL (1964) an Dipurena reesi unternahm, führten, in Übereinstimmung mit Resultaten von WeILER-STOLT (1960), zu dem Ergebnis, daß undeterminierte interstitielle Zellen (IZellen) der Knospungsregion des Hydranthen die Anlage der Medusenknospen aufbauen, ferner, daß eine Zuwanderung von I-Zellen aus anderen Regionen des Hydranthen nicht erforderlich ist und offenbar auch nicht erfolgt. Entsprechende Untersuchungen zur Entstehung der Blastostyle und Medusenknospen bei Eirene viridula fehlten bislang und sollten nun vorgenommen werden, nachdem dieser Abschnitt des metagenetischen Entwicklungsganges zuverlässig ausgelöst werden konnte.

Das experimentelle Konzept ging von der Annahme aus, daß auch bei Eirene viridula I-Zellen, die sowohl in den Hydranthen, als auch in den Hydrocauli und den Stolonen histologisch nachweisbar sind (Ab. 7a, c), bei der Morphogenese der Blastostyle und Medusenanlagen, in denen sie ebenfalls in großer Zahl gefunden wurden (Abb. 7b), ausschlaggebend sind. Die selektive Ausschaltung der I-Zellen nach der von MüLler (1968) an Hydractinia echinata ausgearbeiteten Methode durch kurzzeitige Inkubation mit alkylierenden Zytostatika mußte nach Umsetzen der so behandelten Stöcke in höhere Temperaturen zu Ausfallreaktionen bei der Bildung der Geschlechtsgeneration führen, wenn unsere Voraussetzungen zutrafen. 


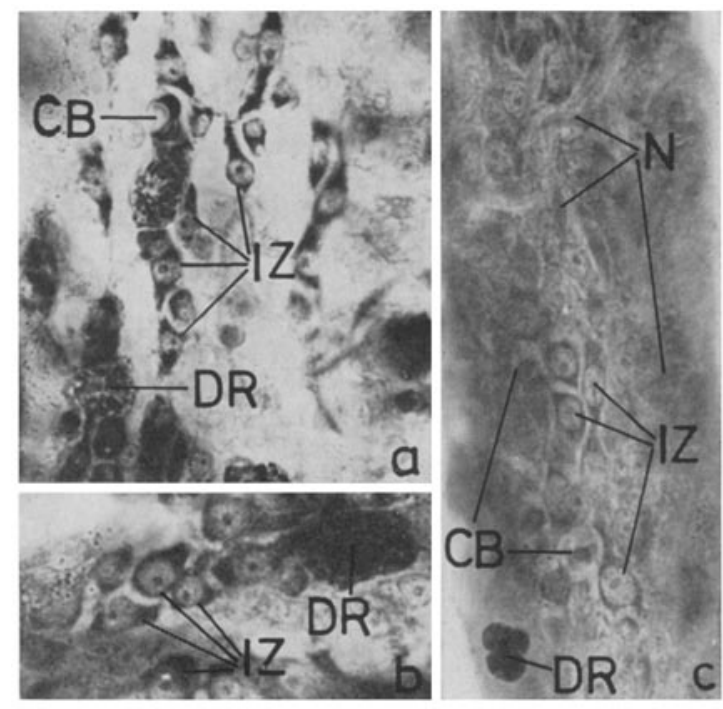

Abb. 7: Eirene viridula. Ausschnittvergrößerungen gefärbter Totalpräparate unbehandelter Stödke. a Polyp (320:1), b Medusenknospe (800:1), c Stolo (800:1)

\section{METHODIK}

Für die erste Versuchsreihe zu diesem Fragenkomplex verwendeten wir das Zytostatikum Trenimon $[=2,3,5$ - Tris-äthylenimino-benzochinon (1.4)]. Jeweils 3 bei $20^{\circ} \mathrm{C}$ herangezogene, freischwimmende Stöcke von Eirene viridula mit einer Ausgangsgröße von 20 Polypen wurden während 10 Minuten durch Eintauchen der Kolonien in Lösungen behandelt, die $4 \times 10^{-2}, 4 \times 10^{-3}$ oder $4 \times 10^{-4} \mathrm{mg}$ Treñmon pro $\mathrm{ml}$ Meerwasser enthielten. Der Behandlungszeitpunkt, bezogen auf die Temperaturerhöhung, wurde gleichfalls variiert um eventuell durch die Temperaturänderung bewirkte Sensibilitätsänderungen gegenüber der Droge erfassen zu können. Je eine Konzentrationsreihe mit $3 \times 3$ Stöcken wurde: (a) vor dem Umsetzen, (b) 24 Std. und (c) $48 \mathrm{Std}$. nach dem Umsetzen von $20^{\circ}$ nach $25^{\circ} \mathrm{C}$ in die Zytostatikumlösung eingebracht. Jeder Stock wurde nach der Behandlung 5 mal je 5 Minuten in $50 \mathrm{ml}$ reinem Meerwasser ausgewaschen. Wasserwechsel erfolgte weiterhin zweimal wöchentlich.

In einer weiteren Versuchsreihe, an Hand derer wir die histologische Uberprüfung der Auswirkungen der TrENIMON-Behandlung mit nachfolgendem Temperaturwechsel vornahmen, wurden je ein freischwimmender und zwei sessile Stöcke mit 30 bis 40 Polypen 10 Minuten in $4 \times 10^{-2}$ oder $4 \times 20^{-3} \mathrm{mg}$ TRenimon $/ \mathrm{ml}$ Meerwasser inkubiert und nach Auswaschen von $20^{\circ}$ nach $25^{\circ} \mathrm{C}$ umgesetzt. Mit denselben Wirkstoffkonzentrationen wurden ferner 2 sessile Stöcke getestet, denen zuvor alle Polypen amputiert worden waren. In Einzelversuchen wurden auch freischwimmende und sessile Stöcke vor dem Temperaturwechsel in Lösungen behandelt, die nur $1 \times 10^{-3} \mathrm{bzw}$. $1 \times 10^{-4} \mathrm{mg}$ Trenimon $/ \mathrm{ml}$ enthielten. Die histologische Auswertung erfolgte an einzelnen, zu verschiedenen Zeiten nach der Zytostatikumeinwirkung und Temperatur- 
änderung aus den Stöcken entnommenen Stoloabschnitten mit oder ohne Polypen, die nach Fixierung und Stïckfärbung als Totalpräparate in EukITT eingedeckt wurden. Fixierung: BouIN (in Meerwasser angesetzt); Färbung: Panoptische Färbung nach Pappenheim (RomeIs 1948, $\$ 1403$ ). Diese Färbtechnik hatte sich bei verschiedenen Hydroiden-Spezies zum Nachweis interstitieller Zellen bewährt (vgl. MüLLER 1964).

\section{Ergebnisse der Bebandlung mit dem Zytostatikum TRENIMON}

24 Stunden nach Trenrmon-Behandlung war das Erscheinungsbild der Polypenkolonien noch unverändert, erst nach 48 Stunden traten teilweise Veränderungen an den Polypen auf. Sie führten bei den mit der höchsten Trenimon-Konzentration $\left(4 \times 10^{-2} \mathrm{mg} / \mathrm{ml}\right)$ behandelten Stöcken zum Verlust aller Polypen bis zum 4 . Tag und zuweilen zur Fragmentation des Stologewebeschlauches innerhalb des Peridermrohres.

Während der folgenden Tage wuchsen aus zahlreichen Hydrocauli eingeschmolzener Polypen stolonenartige Gebilde mit Peridermhülle und einem Gewebeschlauch mit Ektoderm- und Entodermanteil aus, die bei sessilen Stöcken gelegentlich zum Substrat hinwuchsen und mit den Stolonen fusionierten. Polypenneubildung erfolgte nicht, ebensowenig bei dem Stock, dessen Polypen zuvor entfernt worden waren. In der nächst niedrigeren Trenimon-Konzentration $\left(4 \times 10^{-3} \mathrm{mg} / \mathrm{ml}\right)$ behandelte Kolonien zeigten diese extreme Reaktion nur in einem Fall. Bei den übrigen erfolgte zwar Tentakeleinschmelzung und Absterben einiger Polypen (eine Kolonie verlor sogar alle Hydranthen), jedoch war nach 15 Tagen wieder Polypenneubildung und nach 17 Tagen sogar vereinzelt Bildung von Medusenknospen zu beobachten. Bei den beiden sessilen Stöcken dieser Behandlungsstufe wuchsen die Hydrocauli zahlreicher eingeschmolzener Polypen später zum Mehrfachen der iblichen Länge aus, ohne die Anlage von Polypenköpfchen zu zeigen; überdies war die Polypendegression erst in der 4. Woche nach Versuchsbeginn zum Stillstand gekommen. Bei einigen Polypen entstandene, als Blastostylanlagen angesehene Bildungen entwickelten sich stolonenartig weiter. Der Stock, dessen Polypen vor der Behandlung amputiert worden waren, bildete keine neuen Polypen aus; das Stologewe ebe degenerierte innerhalb von 4 Wochen.

Nach der Behandlung in der niedrigsten Konzentration ( $4 \times 10^{-4} \mathrm{mg}$ TRENIMON/ ml) vor dem Temperaturwechsel veränderten sich die Polypen morphologisch nicht. Es traten Medusenknospen zum selben Zeitpunkt wie bei unbehandelten Stöcken auf. Zu einem späteren Zeitpunkt als erwartet lösten sich auch freie Medusen ab; sie waren kleiner als solche von Kontrollstöcken und überdies deformiert. Behandlung 24 oder 48 Std. nach Temperaturerhöhung bewirkte Verzögerung des Beginns der Medusenknospung - bezogen auf den Zeitpunkt des Temperaturwechsels - um einen Tag gegenüber der Mehrzahl nicht behandelter Kolonien; die zuerst freigesetzten Medusen waren ebenfalls deformiert.

Aus den Befunden war ersichtlich, daß TrenImon in der angewandten Dosierung von $10 \mathrm{~min} \times 4 \times 10^{-2} \mathrm{mg} / \mathrm{ml}$ die Polypenstöcke nicht nur kurzfristig und reversibel schädigte, wobei die Kolonien Rückbildungsstadien durchliefen, wie sie auch von KARBE (1972) nach Intoxikation mit Schwermetallverbindungen gefunden und klassifiziert worden waren, sondern sie auf Dauer entscheidender morphogenetischer Poten- 
zen beraubte: Polypenbildung und Medusenknospung waren unterbunden, Entwicklung fand nur auf der Differenzierungsstufe von Stolonen statt (Abb. 8a). Auch Inkubation in der nächst geringeren Konzentration $\left(4 \times 10^{-3} \mathrm{mg} / \mathrm{ml}\right)$ bewirkte bei den sessilen Stöcken nach teilweiser Polypenreduktion "Stolonisation“ von Hydrocauli eingeschmolzener Polypen und Polypenanlagen sowie der - der Topographie zufolge vermuteten - Blastostylanlagen. Erst bei Anwendung noch geringerer TRenimoN-Dosen $\left(4 \times 10^{-4}, 1 \times 10^{-4} \mathrm{mg} / \mathrm{ml}\right)$ waren Stockentwicklung und Medusenknospung nurmehr geringfügig gehemmt, nicht aber erheblich verändert.

Die Untersuchung gefärbter Totalpräparate ergab, daß 3 Tage nach TrENIMONBehandlung mit Dosen von $4 \times 10^{-2}$ oder $4 \times 10^{-3} \mathrm{mg} / \mathrm{ml}$ in den verbliebenen Hydrocauli und Stolonen keine als typische interstitielle Zellen ansprechbare Zellen mehr

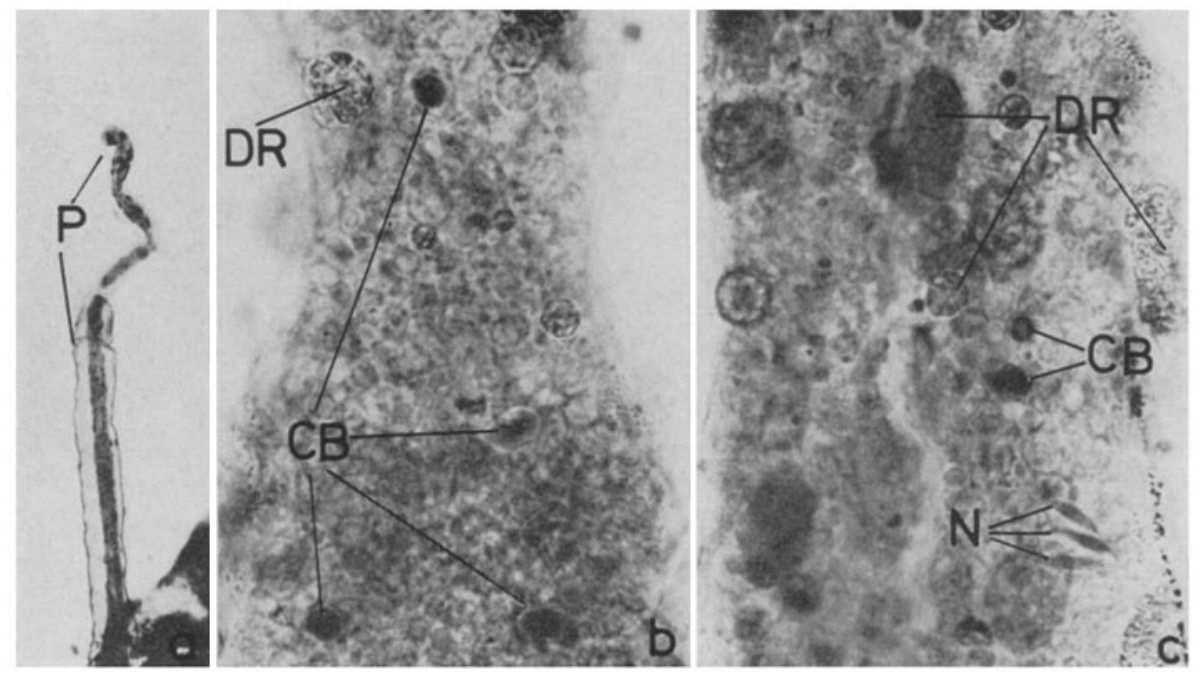

Abb. 8: Eirene viridula. Ausschnittvergrößerungen gefärbter Totalpräparate TRenrmon-behandelter Stöcke. a aus Hydrocaulus entstandenes, stolonenartiges Gebilde $(63: 1)$ (8. Tag nach Behandlung mit $\left.4 \times 10^{-2} \mathrm{mg} / \mathrm{ml}\right) ; b$ und $c$ I-Zellen-freie Stolonen (800:1) (5. Tag aach Behandlung mit $4 \times 10^{-2} \mathrm{mg} / \mathrm{ml}$ )

nachweisbar waren. Es fanden sich im nunmehr stark eosinophilen Gewebe Epithelzellen, Drüsenzellen, Nematocysten, Cnidoblasten mit weit fortgeschrittener Kapselbildung und zahlreiche pyknotische Zellen mit unterschiedlichen Durchmessern. Die Lumina der Entodermkanäle waren häufig mit Zellmaterial angefüllt. Wir schlossen. daraus, daß Trenimon in diesen Konzentrationen sowohl die I-Zellen als auch die jungen Cnidoblasten angriff. Da bis zum 13. Tag das zytologische Bild (Abb. 8b, c) im wesentlichen unverändert blieb, mußten wir eine irreversible Schädigung dieser Zelltypen annehmen. Die oben geschilderte Ausbildung stolonenartiger Schläuche, die histologisch alle Charakteristika von Stolonengewebe zeigten (Abb. 8a) war somit allein auf die morphogenetische Potenzen des Ektoderms und Entoderms zurückzuführen; in den Knospenregionen gefundene Mitosestadien stützen diese Deutung. Daraus ist abzu- 
leiten, daß für Entwicklungsleistungen oberhalb der stolonialen Differenzierungsstufe, also Polypenausbildung und Medusenknospung, I-Zellen vonnöten sind. Diese Auffassung wird durch den Befund erhärtet, daß Polypenkolonien, die in den niedrigsten Trenimon-Konzentrationen $\left(4 \times 10^{-4} ; 1 \times 10^{-4} \mathrm{mg} / \mathrm{ml}\right)$ behandelt worden waren und danach Stockvergrößerungen und Medusenknospung zeigten, zwar ebenfalls 3 Tage nadh Inkubation mit der Droge pyknotische Zellen aufwiesen, jedoch zu diesem Zeitpunkt und auch während der gesamten Beobachtungsdauer I-Zellen besaßen

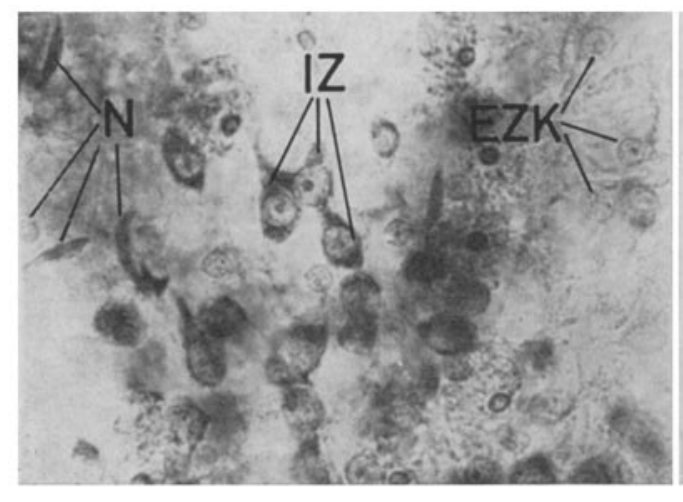

a

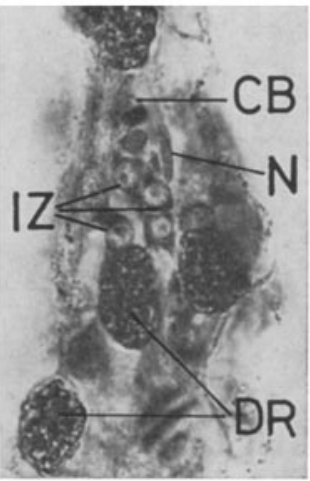

$\mathrm{b}$

Abb. 9: Eirene viridula. Ausschnittvergrößerungen gefärbter Totalpräparate TREnımon-behandelter Stöcke. a Hydranth eines restituierten, freischwimmenden Stockes $(800: 1)$ (20. Tag nach Behandlung mit $4 \times 10^{-3} \mathrm{mg} / \mathrm{ml}$ ); $b$ Stolo eines morphologisch nicht exkennbar geschädigten Stockes (800:1) (10. Tag nadh Behandlung mit $1 \times 10^{-4} \mathrm{mg} / \mathrm{ml}$ ). Abkürzungen in den Abbildungen 7-9: $\mathrm{CB}=$ Cnidoblast; $\mathrm{DR}=$ Drüsenzelle; $\mathrm{EZK}=$ Epithelzellkern; IZ = Interstitielle Zellen; $N=$ Nematocyste; $P=$ Periderm. Alle Präparate wurden in BouxN fixiert und mit der Panoptischen Färbung nach Pappenherm gefärbt

(Abb. 9b). Zumindest während der ersten Tage erschien deren Zahl aber deutlich verringert. Nidht eindeutig geklärt werden konnten die Verhältnisse bei einem Stock, der nach Behandlung mit $4 \times 10^{-3} \mathrm{mg}$ Trenimon $/ \mathrm{ml}$ zwar alle Polypen verlor, jedoch nach 16 Tagen wieder Hydranthen sproßte und auch Medusenknospen anlegte, und bei dem auch dann, anders als in den Stolostichproben vom 5 . +8. Tag, I-Zellen in groBer Zahl aufgefunden wurden. Wir deuten den Fall dahingehend, daß bei diesem Stock zumindest einige I-Zellen nicht oder jedenfalls nicht irreversibel durch das Agens geschädigt wurden, die sich nach einer Latenzzeit wieder vermehrten und erneute Stockpropagation ermöglichten. Wir halten es für weniger wahrscheinlich, daß I-Zellen auf dem Wege über eine Umdifferenzierung von Epithelzellen entstanden waren, wie dies von Davis (1970) bei Entodermexplantaten von $H y d r a$ beschrieben wurde.

\section{DISKUSSION UND SCHLUSSFOLGERUNGEN}

Die vorgenommenen Untersuchungen zur Stodkentwicklung von Eirene viridula unter konstanten Temperaturbedingungen sowie nach positivem oder negativem Tem- 
peraturwechsel berücksichtigten zwei Aspekte: (1) Stockvergrößerung durch Polypensprossung und (2) Bildung von Medusenknospen und Medusen.

Wir stellten fest, daß die Mehrzahl der sessilen Stöcke am Ende der 17tägigen Beobachtungszeit höhere Polypenanzahlen erreichten als freischwimmende Stöcke derselben Ausgangsgrößenklassen; dies traf sowohl für die bei $20^{\circ} \mathrm{C}$ gehaltenen als auch für die dem positiven Temperaturwechsel von $20^{\circ}$ nach $25^{\circ} \mathrm{C} \mathrm{bzw}$, nach $29^{\circ} \mathrm{C}$ unterworfenen Kolonien zu. Ein Grund für die unterschiedliche Zunahme der Polypenanzahlen könnte darin gesucht werden, daß bei freischwimmenden Stöcken der Anteil der Stolonen an der Stodkmasse höher ist als bei der sessilen Form. Auch wurde beobachtet, daß bei großen freischwimmenden Stöcken Hydrocauli und Hydranthen zu größerer Länge auswuchsen. Beide Faktoren dürften aber bei den $z$. T. sehr kleinen Stöcken, die in unseren Versuchen verwendet wurden, kein erhebliches Gewicht gehabt haben. Wir müssen vielmehr davon ausgehen, daß der permanente Untergrundkontakt bei den sessilen Stöcken wachstumsfördernd wirkt. Hierfür sprechen auch an isolierten Stolostücken von Dipurena gewonnene Ergebnisse, bei denen Polypenknospung durch Verhindern der Festsetzreaktion gehemmt werden konnte (GÄRTNER, persönliche Mitteilung). Entgegen unserer Erwartung war bei beiden Wuchsformen der Stockzuwachs nach Umsetzen in $25^{\circ}$ oder $29^{\circ} \mathrm{C}$ nicht generell höher als bei den Kolonien der $20^{\circ} \mathrm{C}$ Stufe, sondern in 50 bis $77 \%$ der Fälle sogar kleiner. Ahnliche Befunde beschrieben auch KINNE (1956) für Cordylopbora caspia, bei der in vergleichbaren Salzgehaltsstufen bei ca. $23^{\circ} \mathrm{C}$ ein geringerer Stockzuwachs erfolgte als bei $16^{\circ} \mathrm{C}$, und CROwELI (1957) für Campanularia, bei der ebenfalls bei $22^{\circ} \mathrm{C}$ eine geringere Polypenpropagation zu beobachten war als bei $16^{\circ} \mathrm{C}$. Als eine Ursache der resultierenden geringeren Stockendgröße konnte Stockdegression am 9. Tag nach Temperaturwechsel nachgewiesen werden, wobei wir den Eindruck gewannen, daß vorwiegend die älteren Polypen resorbiert wurden. Als weitere Erklärungsmöglichkeit für die geringere Polypenzunahme bei erhöhten Temperaturen bleibt die zusätzliche Bildung von Blastostylen und Medusenknospen zu diskutieren. GüNzl (1964) postulierte für Dipurena reesi im Sinne der Theorie von SpIEgelmann (1945) eine "physiologische Konkurrenz" zwischen Polypen- und Medusenknospung, bei der in kleineren Stöcken die Polypensprossung dominierte und erst bei nachlassender Polypenbildung sich die Medusenknospung durchsetzte. Als verantwortlich für die Rückbildung früh angelegter Medusenknospen wurde ein von den Hydranthen ausgehender Hemmstoff angesehen.

Übertragen auf den Fall von Eirene viridula kann festgestellt werden, daß bei kleinen sessilen Stöcken ebenfalls relativ stärkeres Koloniewachstum und geringere Medusenbildungstendenz koinzidierten, während bei den im Stockwachstum gehemmten, freischwimmenden Stöcken die Zahl der medusenknospenbildenden Stöcke wesentlich erhöht war. Dies weist auf eine Konkurrenzsituation der beiden Knospentypen auch bei Eirene viridula hin. Der von uns versuchte rechnerische Nachweis dieses „Konkurrenzeffektes" durch Summation der Hydranthenzahl bei Versuchsende, der Zahl der mutmaßlich durch Degression ausgefallenen Polypen und der Anzahl der gebildeten Medusenknospen (unter Gleichsetzung des Aufwandes für Polypen- und Medusenknospung) zu einer "Gesamtknospungsaktivität" gelang zwar für einige Stöcke und führte im Vergleich mit den entsprechenden bei $20^{\circ} \mathrm{C}$ aufgezogenen Kolonien zu der erwarteten höheren Knospungsaktivität; eine Gesetzmäßigkeit kann jedoch aus unseren 
Ergebnissen nicht hergeleitet werden. Erschwerend für die Aufstellung einer derartigen Beziehung sind zahlreiche, schwerabschätzbare Fehlermöglichkeiten, vor allem bei der Ermittlung der Anzahl der durch Degression ausgefallenen Polypen und bei der Beurteilung der Medusenknospenzahl. Außerdem steht der SpIEgelmannschen Interpretation die Tatsache entgegen, daß Stöcke, die im Temperaturwechselversuch keine Medusenknospen gebildet hatten - also der "physiologischen Konkurrenz" in diesem Sinne nicht unterlagen - in ihrer Mehrzahl keine höheren Hydranthenzahlen als die bei $20^{\circ} \mathrm{C}$ gehaltenen Kolonien erreichten; eine entsprechende Vermutung, die sich bei einigen kleinen Stöcken des $25^{\circ} \mathrm{C}$-Umsetzversuches ergeben hatte, ließ sich für andere Größenklassen und für den $29^{\circ} \mathrm{C}$-Umsetzversuch insgesamt nicht bestätigen. Es ergab sich ferner, daß auch unabhängig vom Eintreten oder Nichteintreten der Medusenknospung Stodkdegression erfolgte.

Nach Temperaturwechsel von $25^{\circ}$ nach $29^{\circ} \mathrm{C}$ war allgemein ein geringeres Stockwachstum als bei den anderen Versuchen mit positiver Temperaturänderung zu bemerken, allerdings ohne Stagnations- oder Degressionsphasen. Negativer Temperaturwechsel wirkte hemmend oder sogar schädigend auf die Stockentwicklung ein; der Stockzuwachs war, teilweise nach Degression, stets deutlich geringer als bei Kolonien vergleichbarer Ausgangsgröße der $20^{\circ} \mathrm{C}-\mathrm{Stufe}$.

Übereinstimmend mit GüNzL $(1959,1964)$ stellten wir positiven Temperaturwechsel von $20^{\circ}$ auf $25^{\circ}$ oder $29^{\circ} \mathrm{C}$ als auslösenden Faktor für die Bildung von Medusenknospen und Medusen fest. Allerdings zeigte eine Versuchsreihe, daß negativer Temperaturwechsel von $29^{\circ}$ auf $25^{\circ} \mathrm{C}$ ebenso wie der reziproke Versuche bei freischwimmenden Stöcken die Ausbildung der Geschlechtsgeneration bedingen konnte. Allgemein wurde bei freischwimmenden Kolonien, die allerdings nur unter experimentellen Bedingungen, nicht aber unter natürlichen Verhältnissen von Bedeutung sind, eine weit stärkere Reaktion auf den positiven Temperaturwechsel beobachtet - gemessen an der Zahl medusenknopsenbildender Stöcke - als bei den sessilen Kolonien. In dieser Beziehung ergab sich eine gewisse Analogie zu den von CROwerc (1957) und GüNzL (1964) diskutierten Befunden von HaUENSCHILD (1954) an Hydractinia sowie von GRELL an Obelia; bei beiden Arten waren bereits an sehr kleinen, im weiteren Wachsturn experimentell gehemmten Stöcken Gonophoren aufgetreten.

Gonangienstände entwickelten sich ausschließlich an den Hydrocauli ausdifferenzierter Hydranthen unterhalb der Basis der Polypenköpfchen; an ihnen entstanden stets nur Medusenknospen, nie aber Knospen, die sich zu irgendeinem Zeitpunkt zu Polypen weiterentwickelten.

Ob sich der Temperatureinfluß unmittelbar auf die Knospungsregion auswirkt, oder ob - wie von BRÄNDLE (1971) an Podocoryne carnea gezeigt - vom Hypostom (oder einer anderen Region des Hydranthen) ein die Knospung aktivierender Stoff erzeugt werden muß, ist nicht bekannt.

Nicht bestätigen konnten wir die Erfahrungen GüNzLs in bezug auf die Mindestgröße, die ein Stock erreicht haben mußte, bevor er im Temperaturwechselversuch Medusenknospen zu bilden vermochte. Wie dargelegt, zeigten in unseren Experimenten freischwimmende Stöcke ab (A) 1, sessile Stöcke ab (A) 3 ohne vorherigen Zuwachs Medusenknospen.

GüNZL (1964) postulierte in diesem Zusammenhang einen "Größefaktor", der 
nach Vereinzelung aus sehr großen Stöcken in den Ablegerstöcken noch wirksam sein und die Medusenbildung beeinflussen soll. Wir glauben, diesem „Größefaktor" in unseren Versuchen deshalb geringe Bedeutung beimessen zu dürfen, weil wir Versuchsstöcke benutzten, die in zwei Phasen vereinzelt worden waren: Nach der Vereinzelung aus Stammkulturen wuchsen die Stöcke bis zur Größe von ca. 50 Polypen heran und wurden dann gemäß dem Versuchsplan in ihrer Größe reduziert und in die Versuche eingesetzt. Das Problem des "Größefaktors" wäre jedoch nur dadurch zu umgehen, daß für die Temperaturversuche Stöcke verwendet würden, die nicht durch Vereinzelung aus größeren Kolonien, sondern aus Aufzuchten einzelner, direkt aus Planulae entstandenen Primärpolypen gewonnen wurden. Dies war bislang nicht möglich, da Aufzucht der Medusen und geschlechtliche Fortpllanzung unter Laborbedingungen noch nicht erzielt werden konnte.

In Günzls sog. Dauerkulturen traten bei $29^{\circ} \mathrm{C}$ zuweilen spontan Medusenknospen auf. Unsere Versuche ließen nicht sicher erkennen, ob die im Verlauf längerer Anzucht noch entstandenen Medusenknospen in ursächlichem Zusammenhang mit dem zurüdkliegenden Temperaturwechsel standen oder ob es sich bereits um „spontan“ gebildete Knospen handelte. Nach unseren Erfahrungen kommt für die Beurteilung dieser Frage allgemein erschwerend hinzu, daß vor allem freischwimmende Eirene-Kolonien nach längerer Aufzucht bei $20^{\circ} \mathrm{C}$ zuweilen bereits auf kurzfristige, kleine Temperaturerhöhungen von 1,5 bis $2^{\circ} \mathrm{C}$ mit Knospenbildung reagieren können. Wir hatten aus diesem Grunde der Gewährleistung der Temperaturkonstanz besondere Aufmerksamkeit geschenkt.

Die Veränderung der Stockgrößen, bestimmt durch die Aufzählung der ausdifferenzierten Polypen, ließ sich bei den von uns verwendeten Größenklassen ziemlich leicht verfolgen, wobei die Möglichkeit geringfügiger Zählfehler bei den oberen Größenklassen freischwimmender Kolonien naturgemäß etwas größer war als bei den sessilen Kolonien. Die Auslösung der Medusenbildung in den Temperaturwechselversuchen konnte an Hand der Zahl der Medusenknospen bzw. Medusen bildender Stödke ebenfalls gut erfaßt werden; dagegen blieb die quantitative Auswertung der Medusenknospenproduktion ungleich problematischer. Unter den gewählten Bedingungen entwickelte sich nur ein kleiner 'Teil der Medusenknospen zu reifen, sich vom Stodk ablösenden Medusen weiter, alle anderen atrophierten bzw. wurden resorbiert.

Unter Berücksichtigung der unterschiedlichen Entwicklungsdauer der Knospen war es ohne weiteres denkbar, daß beispielsweise an zwei aufeinanderfolgenden Tagen am selben Gonangienstand registrierte Medusenknospen nicht einer, sondern zwei verschiedenen "Generationen " von Medusenknospen angehörten, ohne daß dies zahlenmäßig in Erscheinung treten würde. Auf der Basis solcher Zählungen, die bei allen Versuchsreihen vorgenommen wurden, würden Angaben über die während des Versuchszeitraumes produzierten Medusenknospen nicht unerhebliche Fehler enthalten, die sich nicht eliminieren lassen. Wir mußten deshalb auf die Vorlage und Erörterung dieser Zahlenwerte verzichten.

Die Bedeutung der I-Zellen für die Medusenknospung und auch für die Polypensprossung konnte durch die Behandlung mit dem alkylierenden Zytostatikum TreNIMON, kombiniert mit positivem Temperaturwechsel, aufgezeigt werden. Übereinstimmend mit den Ergebnissen von MüLLER (1968) an Hydractinia konnte nach Inkubation 
mit $4 \times 10^{-2} \mathrm{mg}$ Trenimon $/ \mathrm{ml}$ während 10 Minuten die Ausschaltung der I-Zellen (und sehr wahrscheinlich auch der jungen Cnidoblasten) festgestellt werden. Nach den Folgerungen MüLLERs aus Experimenten mit verschiedenen Zytopharmaka kommt diese Wirkung durch die endgültige Zerstörung der RNS-Syntheseapparates und ihres Steuerungssystems, einschließlich der DNS-Matrize, zustande. Nach dieser Behandlung vermochten die Eirene-Stöcke weder Polypen zu propagieren, noch Medusenknospen zu bilden, sondern nur noch Entwidklung auf der stolonialen Differenzierungsstufe zu vollziehen. Der Vergleich zeigt, daß I-zellenfreie Stöcke von Hydractinia noch größere morphogenetische Potenzen besaßen: sie entwickelten innerhalb der etwa sechswöchigen Uberlebenszeit sogar Polypen. Nach Trenrmon-Einwirkung in mittleren und hohen Dosen erfolgte stets auch eine tiefgreifende, morphologische Stockschädigung; dauernde Ausschaltung der I-Zellen bei gleichzeitiger Erhaltung der Polypen wurde in unseren Versuchen nicht beobachtet. Wir sehen jedoch die Polypenreduktion eher als unspezifische Komponente der Trenimon-Wirkung an, da dieser Effekt auch mit anderen Mitteln, z. B. durch Intoxikation mit Schwermetallen (KARBE 1972), hervorgerufen werden konnte und auch nach Temperaturänderungen auftrat.

\section{ZUSAMMENFASSUNG}

1. Die Entwicklung sessiler und freischwimmender Stöcke verschiedener Größenklassen von Eirene viridula (Thecata-Leptomedusae) wurde unter verschiedenen Temperaturbedingungen untersucht.

2. Sowohl nach Aufzucht bei konstant $20^{\circ} \mathrm{C}$ als auch nach positivem Temperaturwechsel von $20^{\circ}$ nach $25^{\circ}$ oder $29^{\circ} \mathrm{C}$ lag die Endgröße der Mehrzahl der sessilen Stöcke höher als die der freischwimmenden Kolonien.

3. Die diesem Temperaturwechsel unterworfenen sessilen und freischwimmenden Stöcke waren bei Versuchsende in der Mehrzahl kleiner als die der $20^{\circ} \mathrm{C}-\mathrm{Stufe}$. Für diese Zuwachsminderung ist teilweise die am 9. Tag nach Temperaturerhöhung beobachtete Resorption von Polypen verantwortlid; als weiterer Faktor wird die "physiologische Konkurrenz" der Polypenpropagation mit der durch den Temperaturwechsel ausgelösten Medusenbildung diskutiert.

4. Negativer Temperaturwechsel wirkte sich im ganzen hemmend, in einigen Fällen sogar ausgesprochen schädigend auf die Stockentwicklung aus.

5. Positiver Temperaturwechsel von $20^{\circ}$ nach $25^{\circ}$ oder $29^{\circ} \mathrm{C}$ löste bei Stöcken beider Wuchsformen die Bildung von Gonangienständen mit Blastostylen und Medusenknospen aus. Ausgenommen bei den nach $25^{\circ} \mathrm{C}$ umgesetzten sessilen Stöcken entwickelten sich stets einige der Medusenknospen zu voll ausdifferenzierten, sich vom Stock ablösenden Medusen. Die übrigen Medusenknospen atrophierten. Eine Umentwicklung der Medusenknospen zu Polypen wurde nie beobachtet.

6. Positiver Temperaturwechsel von $25^{\circ}$ nach $29^{\circ} \mathrm{C}$ löste lediglich bei freischwimmenden Kolonien Medusenknospenbildung aus; bei wenigen Stöcken lösten sich auch Medusen ab.

7. Freischwimmende Stöcke reagierten auf positive Temperaturänderung mit Bildung 
von Medusenknospen in der Mehrzahl 1 bzw. 2 Tage eher als sessile Stöcke, bei letzteren war auch die Knospungsrate insgesamt geringer.

8. Negativer Temperaturwechsel (von $25^{\circ}$ nach $20^{\circ}, 29^{\circ}$ nach $20^{\circ}$ und $29^{\circ}$ nach $25^{\circ} \mathrm{C}$ führte nur im Umsetzversuch von $29^{\circ}$ nach $25^{\circ} \mathrm{C}$ bei freischwimmenden Kolonien, nicht aber bei sessilen Stöcken, zur Bildung von Medusenknospen und bei einigen der Stöcke auch von freien Medusen.

9. Für die Auslösung der Medusenknospung war keine bestimmte Mindestgröße nachweisbar. Medusenknospen entstanden nach positiver Temperaturänderung bei freischwimmenden Stödken ohne vorherigen Stockzuwachs bereits ab Ausgangsgröße (A) 1, freie Medusen ab (A) 3. Bei sessilen Stöcken traten Medusenknospen ebenfalls ohne vorherige Stockvergrößerung ab (A) 3 auf.

10. Inkubation mit dem alkylierenden Zytostatikum Trenrmon in Verbindung mit Temperaturerhöhung von $20^{\circ}$ auf $25^{\circ} \mathrm{C}$ führte bei der Einwirkung von $4 \times 10^{-2} \mathrm{mg} / \mathrm{ml}$ während 10 Minuten in allen Fällen, von $4 \times 10^{-3} \mathrm{mg} / \mathrm{ml}$ bei einem der Stöcke zur vollständigen, irreversiblen Polypenresorption. Weiterentwicklung erfolgte nur auf der Differenzierungsstufe von Stolonen. Die übrigen mit $4 \times 10^{-3} \mathrm{mg} / \mathrm{ml}$ und alle mit $4 \times 10^{-4} \mathrm{mg} / \mathrm{ml}$ TrENIMON behandelten Stöcke vermochten - teilweise erst nach Überwinden einer Degressionsphase - Polypen zu sprossen und in die Medusenbildung einzutreten.

11. Behandlung mit $4 \times 10^{-2} \mathrm{mg} / \mathrm{ml}$ Trenimon bewirkte irreversible Schädigung und den Verlust aller I-Zellen. Nach Behandlung mit nur $1 \times 10^{-3}$ oder $1 \times 10^{-4} \mathrm{mg} / \mathrm{ml}$ blieben I-Zellen erhalten. Aus den Befunden wurde deshalb geschlossen, daß I-Zellen für die Morphogenese von Polypen und Medusen bei Eirene viridula unabdingbar sind.

Danksagungen. Die Unterstïtzung dieser Arbeit durch die Deutsche Forschungsgemeinschaft war durch Sachbeihilfen an Dr. D. K. HOFMann gegeben. Den BAYER-Werken danken wir für die Uberlassung des für unsere Untersuchungen benötigten Zytostatikums Trenimon. Für die Durchführung der Erhaltungszuchten und für die Ausführung der Abbildungsvorlagen sind wir Frau F. LABussière sehr zu Dank verpflichtet.

\section{ZITIERTE LITERATUR}

BRÄNDLE, E., 1971. Bedeutung der kolonialen Komponenten für die Bildung und Differenzierung der Medusen von Podocoryne carnea. Wilhelm Roux Arch. EntwMech. Org. 166, $254-286$.

CROwELL, S., 1957. Differential responses of growth zones to nutritive level, age, and temperature in the colonial hydroid Campanularia. J. exp. Zool. 134, 63-90.

Davis, L. E., 1970. Further observations on dividing and non-dividing cnidoblasts in the regenerating gastrodermis of Hydra. Z. Zellforsch. mikrosk. Anat. 105, 526-537.

GüNZL, H., 1959. Zur Physiologie der Medusenbildung bei Eirene viridula. Naturwissenschaften $46,337$.

- 1964. Untersuchungen über die Auslösung der Medusenknospung bei Hydroidpolypen. Zool. Jb. (Anat. Ontogenie Tiere) 81, 491-528.

HaueNSCHXLD, C., 1954. Genetische und entwicklungsphysiologische Untersuchungen über Intersexualität und Gewebeverträglichkeit bei Hydractinia echinata. Wilhelm Roux Arch. EntwMech. Org. 147, 1-41. 
Karbe, L., 1972. Marine Hydroiden als T'estorganismen zur Prüfung von Abwasserstoffen. Die Wirkung von Schwermetallen auf Kolonien von Eirene viridula. Mar. Biol. 12, 316-328.

KrnNe, O, 1956. Uber den Einfluß des Salzgehaltes und der Temperatur auf Wachstum, Form und Vermehrung bei dem Hydroidpolypen Cordylophora caspia. Zool. Jb. (Allg. Zool. Physiol. Tiere) 66, 565-638.

MüLter, W. A., 1964. Experimentelle Untersuchungen über Stockentwicklung, Polypendifferenzierung und Sexualchimären bei Hydractinia echinata. Wilhelm Roux Arch. EntwMech. Org. 155, 181-268.

- 1967. Differenzierungspotenzen und Geschlechtsstabilität der I-Zellen von Hydractinia ecbinata. Wilhelm Roux Arch. EntwMech. Org. 159, 412-432.

- 1968. Elimination der I-Zellen durch alkylierende Cytostatika und deren Effekte auf die Embryonalentwicklung bei Hydractinia echinata. Expl. Cell Res. 49, 448-458.

ReIsinger, E., 1957. Zur Entwicklungsgeschichte und Entwicklungsmechanik von Craspedacusta (Hydrozoa, Limnotrachilina). Z. Morph. Okol. Tiere 45, 656-698.

Romers, B., 1948. Mikroskopische Technik. Oldenbourg Verl., München. 695 pp.

Spiegrlanan, S., 1945. Physiological competition as a regulatory mechanism in morphogenesis. Q. Rev. Biol. 20, 121-146.

WeItER-STolt, B., 1960. Über die Bedeutung der interstitiellen Zellen für die Entwicklung und Fortpflanzung mariner Hydroiden. Wilhelm Roux Arch. EntwMech. Org. 152, 398-455.

Werner, B., 1958. Die Verbreitung und das jahreszeitliche Auftreten der Anthomeduse Ratbkea octopunctata sowie die Temperaturabhängigkeit ihrer Entwicklung und Fortplanzung. Helgoländer wiss. Meeresunters. 6, 160-170.

- 1961. Morphologie und Lebensgeschichte sowie Temperaturabhängigkeit der Verbreitung und des jahreszeitlichen Auftretens von Bougainvillia superciliaris. Helgoländer wiss. Meeresunters. 7, 230-237.

- 1968. Polypengeneration und Entwicklungsgeschichte von Eucbeilota maculata (ThecataLeptomedusae). Mit einem Beitrag zur Methodik der Kultur mariner Hydroiden. Helgoländer wiss. Meeresunters. 18, 136-168.

Anschrift des erstgenannten Autors: Studienrat M. Bierbach

Institut für Entwicklungsphysiologie

Universität Köln

5 Köln-Lindenthal

Gyrhofstraße 17

Bundesrepublik Deutschland 